\title{
Synergies in control of the cutting processes
}

\author{
Vilor Zakovorotny, and Valery Gvindjiliya* \\ Don State Technical University, 344000 Rostov-on-Don Gagarin sq. 1, Russian Federation
}

\begin{abstract}
As the rule metal-cutting machine have controllable movements of the executive tool from the computer. However, the shape-generating movements differ from the executive elements movements. These differences are defined by the influence of the deformation of the tool and detail. The deformations are conditioned by complex nonlinear transformation in all system. In turn, the process dynamic system is determined on the basis of the disclosure of the dynamic subsystem interaction. While the dynamic link parameters depend on the trajectories of the executive elements movement. Therefore, it is necessary to agree on the trajectories of the executive elements and the cutting dynamic properties. Agreement characterize the synergies of the external control with the internal links, it characterize the next stage of the enhancement of the cutting processing control.
\end{abstract}

\section{Introduction}

After the publishing of the I. Prigogin's and G. Haken's works [1 - 4] the many problems of the control in the technical systems have become to consider take into account the synergies approach [5 - 6]. In the monographs [7, 8] showed that the processing on the metal-cutting machine represent the complex system in which all interacting subsystems through the cutting are interrelated. The control in many cases is considered in connection with development of the CNC [9, 10]. The local control systems are studied [11 - 13]. The problem of the agreement of the control and the system properties are considered $[15,15]$. The agreement of the external and internal control is based on the cutting forces causing the deformation displacement and depending on the trajectories of the executive elements. This condition is done not ever. If we following the synergies concept that in addition it is necessary to agree on the external control with the internal properties on criterion of the trajectories asymptotically stable. The article is devoted to solving these problems.

\section{The mathematical model of the controlling dynamic system of the cutting}

The mathematical model of the trajectories transformation of the executive elements of the machine (space $Z=\left\{Z_{1}, Z_{2}, Z_{3}\right\}^{T} \in \mathfrak{R}_{Z}^{(3)}$ ) (fig.1 «a») in the trajectories of the shape-generation

\footnotetext{
* Corresponding author: sinedden@yandex.ru
} 
movement (space $W=\left\{W_{1}, W_{2}, W_{3}\right\}^{T} \in \mathfrak{R}_{W}^{(3)}$ ) is considered. The deformation displacements are formed (point $\left\langle 0 »\right.$ in fig. 1 «a» displace in point $\left\langle 0^{I} »\right)$ by interaction of the tool with detail. In the future to study of the agreement features we will be limited to the case of the manufacture of the absolutely rigid detail. Besides let's consider $Z_{3}=\Omega=$ const. Then the time of the one turnover $T=(\Omega)^{-1}=$ const. The deformations are considered in the coordinate system $Y=\left\{Y_{1}, Y_{2}, Y_{3}\right\}^{T} \in \mathfrak{R}_{Y}^{(3)}$ which originates in the point of the contact of the tool vertex with detail without of the deformation displacement of the tool. Then $W=\left\{Z_{1}+Y_{1}, Z_{2}+Y_{2}, W_{3}=Y_{3}\right\}^{T}$. If the forces $F_{\Sigma}=\left\{F_{1, \Sigma}, F_{2, \Sigma}, F_{3, \Sigma}\right\}^{T}$ are set then the deformations $Y$ depend on the properties of the inertial (matrix $m$ ), the dissipation (matrix $h$ ) and the elastic (matrix $c$ ) of the tool subsystem that is

$$
m \cdot \frac{d^{2} Y}{d t^{2}}+h \cdot \frac{d Y}{d t}+c \cdot Y=F_{\Sigma}
$$

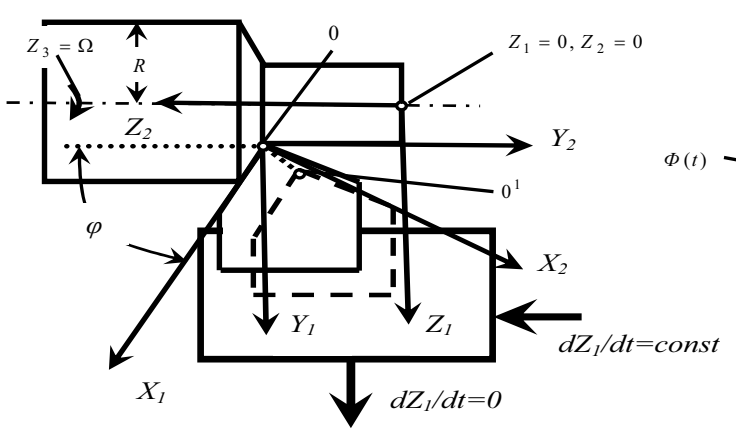

«a»

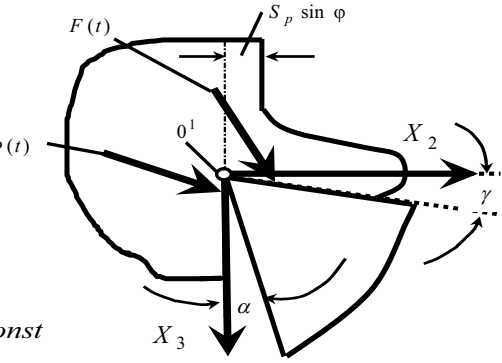

«b»

Fig. 1. Scheme of the formation of the trajectories of the shape-generating movements: $a-$ interrelation of the coordinates of the controlling dynamic cutting system; $b$ - scheme of the formation of the cutting forces

In (1) $c=\left[c_{s, k}\right], s, k=1,2,3$ is the real, symmetric and positive-definite matrix that is potential. This modeling provide the deformation in new coordinate system $\left\{X_{1}, X_{2}, X_{3}\right\}^{T} \in \mathfrak{R}_{X}^{(3)}$ that can be obtained the based on rotation of the system $\mathfrak{R}_{Y}^{(3)}$ with help Euler's corners. It is shown [17, 18], that the axis $Y_{3}$ match with one of the axis of the rigidity ellipsoid. Therefore in plane $Y_{1}, Y_{2}$ we have the rigidity ellipsoid. If corner $\varphi$ combine with the orientation of the rigidity ellipse, then the matrix $c$ becomes the diagonal. If we assume that the matrixes of the inertial $m=\left\lfloor m_{s, k}\right\rfloor, s, k=1,2,3 ; m_{s, s}=m ; m_{s, k}=0, n p u: s \neq k \quad$ and the speed coefficients $h=\left\lfloor h_{s, k}\right\rfloor s, k=1,2,3 ; h_{s, s}=h ; h_{s, k}=0, n p u: s \neq k$ is diagonal with equal coefficients then the most important properties of the system will be disclosure. The returns of the axes don't change the matrix of the inertial and speed coefficients with specified conditions. Therefore in studying of the system (1) it is convenient to provide transition from space $\mathfrak{R}_{Y}^{(3)}$ to $X=\left\{X_{1}, X_{2}, X_{3}\right\}^{T} \in \mathfrak{R}_{X}^{(3)}$. Following the synergetic concept the interaction in the coordinates of the space $\mathfrak{R}_{Z}^{(3)}$ and $\mathfrak{R}_{X}^{(3)}$ is represented. The summary forces are 
represented in the form of $F_{\Sigma}=F+\Phi: F=\left\{F_{1}, F_{2}, F_{3}\right\}^{T}$ the force is formed in the area of the front surface of the tool; $\Phi=\left\{\Phi_{1}, \Phi_{2}, \Phi_{3}\right\}^{T}$ - in the area of the contact its two backsides with detail (fig.1«b»). Let's use the results are received earlier at the forces request [20]. For forces $\Phi$

$$
\left\{\begin{array}{c}
\Phi_{1}=\rho_{0}\left\{\int_{t-T}^{t}\left[V_{2}+d X_{1} / d t \cos \varphi-d X_{2} / d t \sin \varphi\right] d t\right\} \sin \varphi \exp \left[\alpha_{0}\left(V_{2} \cos \varphi+d X_{1} / d t\right)\right] \\
\Phi_{2}=\rho_{0}\left[t_{P}^{(0)}-X_{1} \sin \varphi-X_{2} \cos \varphi\right](\sin \varphi)^{-1} \exp \alpha_{0}\left[\left(V_{2} \sin \varphi+d X_{1} / d t \cos \varphi-d X_{2} / d t \sin \varphi\right]\right. \\
\Phi_{3}=k_{T}\left(\Phi_{1}+\Phi_{2}\right)
\end{array}\right.
$$

where $\rho_{0}$ - the forces are reduction to length of the contact $\left[\kappa_{2} /{ }_{M M}\right] ; \alpha_{0}$ - the coefficient of the force increase ; $k_{T}$ - friction coefficient; $V_{2}=d Z_{2} / d t, t_{P}^{(0)}\left(Z_{2}\right)$ - speed of the longitudinal support and allowance without the elastic deformation in point $Z_{2}$. For forces $F$ are operating to the front surface of the tool fairly

$$
\left\{\begin{array}{c}
T_{1} d F_{1} / d t+F_{1}=\rho \chi_{1}\left\{1+\mu \exp \left[-\zeta\left(V_{3}-d X_{3} / d t\right)\right]\right\}\left[t_{P}^{(0)}-X_{1} \sin \varphi-X_{2} \cos \varphi\right] S_{P}(t) \\
T_{2} d F_{2} / d t+F_{2}=\rho \chi_{2}\left\{1+\mu \exp \left[-\zeta\left(V_{3}-d X_{3} / d t\right)\right]\right\}\left[t_{P}^{(0)}-X_{1} \sin \varphi-X_{2} \cos \varphi\right] S_{P}(t) \\
T_{3} d F_{3} / d t+F_{3}=\rho \chi_{3}\left\{1+\mu \exp \left[-\zeta\left(V_{3}-d X_{3} / d t\right)\right]\right\}\left[t_{P}^{(0)}-X_{1} \sin \varphi-X_{2} \cos \varphi\right] S_{P}(t),
\end{array}\right.
$$

where $T_{i}, i=1,2,3$ - the time constant; $\mu$ - the coefficient is determining ratio of the forces in zone low and high speeds; $\zeta$ - the coefficient are determining decrease of the forces with increase of the speed; $V_{3}$ - cutting speed;

$S_{P}(t)=\int_{t-T}^{t}\left[V_{2}+d X_{1} / d t \cos \varphi-d X_{2} / d t \sin \varphi\right] d t$ - the operator of the formation of the turnover feeding take into account speeds of the elastic deformation displacement.

In the analysis it is reasonable to use the principle of the division of the movement into «fast» and «slow» 6,7$]$. This principle follows from properties of the asymptotic behavior of the solves of the nonlinear differential equations are having low parameter at the higher derivatives $[19,20]$. Physically «slow» motion characterize the trajectories of the machine executive element and «slow» displacement of the equilibrium point. Therefore, the dynamic system equilibrium is considered in the relative frame of the coordinates in which the motions are set by the $\mathrm{CNC}$ program (by $\mathrm{Z}$ trajectories). These trajectories are in limits of the bandwidth of the drivers. The "ffast» motions determine components of the deformation displacement in variations relative to the equilibrium of the subsystem of the «slow» motion. The properties of the subsystem of the «fast» motions depend on the dynamic subsystems of the tool, detail and dynamic link are formed by the cutting process. Therefore, the trajectories of the space $\mathfrak{R}_{Z}^{(3)}$ for subsystem of the «fast» motions are the controlling parameters. It is related with parameters (2) and (3) as follows

$$
t_{P}^{(0)}=R-Z_{1} ; V_{2}=d Z_{2} / d t ; V_{3}=2 \pi R Z_{3}
$$

where $R$ - radius of the detail to cutting zone in considering point axis $Z_{2}$.

In works $[7,8,15]$ the technique of the calculation of the control and the trajectories $Z$ are allowing to provide the setting geometry parameters of the detail take into account the 
elastic deformation is supplied. But the results are fair for case when the subsystem of the «fast» motion is stability. Therefore, it is necessary to agree the external control with dynamic properties of the subsystem «fast» motion.

\section{The providing of the stability}

For analysis of the stability it is necessary to consider the linearized equation in variations. Limiting conditions are $t_{P}^{(0)}=$ const,$V_{2}=$ const,$V_{3}=$ const . This is longitude turning with the constant technical regimes. Then the conditions $d X_{i} / d t=0, \quad X_{i}(t)=X_{i}(t-T), \quad i=1,2,3$ and $S_{P}^{(0)}=\int_{t-T}^{t}\left[V_{2}+d X_{1} / d t \cos \varphi-d X_{2} / d t \sin \varphi\right] d t=V_{2} T=$ const are fair in point is set by the trajectories $Z$. In this case the stationary trajectory is the point of the system equilibrium $X^{*}=\left\{X_{1}^{*}, X_{2}^{*}, X_{3}^{*}, F_{1}^{*}, F_{2}^{*}, F_{3}^{*}\right\}^{T}$ and $X^{*}=$ const . For analysis its stability it is necessary to consider [24] the linearized equation in variations. Let's calculate the linearized equation in variations from (1) - (3) after replacement $X_{i}(t)=x_{i}(t)+X_{i}^{*}, i=1,2,3 \quad$ and $F_{i}(t)=f_{i}(t)+F_{i}^{*}, i=1,2,3$

$$
M \frac{d^{2} z}{d t^{2}}+H \frac{d z}{d t}+C z=0
$$

where $z(t)=\left\{x_{1}(t), x_{2}(t), x_{3}(t), f_{1}(t), f_{2}(t), f_{3}(t)\right\}^{T}$;

$$
\begin{aligned}
& M=\left[\begin{array}{cccccc}
m & 0 & 0 & 0 & 0 & 0 \\
0 & m & 0 & 0 & 0 & 0 \\
0 & 0 & m & 0 & 0 & 0 \\
0 & 0 & 0 & 0 & 0 & 0 \\
0 & 0 & 0 & 0 & 0 & 0 \\
0 & 0 & 0 & 0 & 0 & 0
\end{array}\right] ; \\
& H=\left[\begin{array}{cccccc}
h-\rho_{0} S_{P}^{(0)} \alpha_{0} \exp \left(\alpha_{0} V_{2} \cos \varphi\right) & 0 & 0 & -1 & 0 & 0 \\
-\rho_{0} t_{p, \Sigma} \alpha_{0} \exp \left(\alpha_{0} V_{2} \sin \varphi\right) \operatorname{ctg} \varphi & h+\rho_{0} t_{p, \Sigma} \alpha_{0} \exp \left(\alpha_{0} V_{2} \sin \varphi\right) & 0 & 0 & -1 & 0 \\
-k_{T} \rho_{0}\left\{t_{p, \Sigma} \alpha_{0} \exp \left(\alpha_{0} V_{2} \sin \varphi\right) \operatorname{ctg} \varphi+\right. & k_{T} \rho_{0} t_{p, \Sigma} \alpha_{0} \exp \left(\alpha_{0} V_{2} \sin \varphi\right) & h & 0 & 0 & -1 \\
\left.+S_{P}^{(0)} \alpha_{0} \exp \left(\alpha_{0} V_{2} \cos \varphi\right)\right\} & & & & &
\end{array}\right] ; \\
& H=\left[\begin{array}{cccccc}
\left.S_{P}^{(0)} \alpha_{0} \exp \left(\alpha_{0} V_{2} \cos \varphi\right)\right\} & k_{T} \rho_{0} t_{p, \Sigma} \alpha_{0} \exp \left(\alpha_{0} V_{2} \sin \varphi\right) & h & 0 & 0 & -1 \\
0 & 0 & -\chi_{1} \zeta & T_{1} & 0 & 0 \\
0 & 0 & -\chi_{2} \zeta & 0 & T_{2} & 0 \\
0 & 0 & -\chi_{3} \zeta & 0 & 0 & T_{3}
\end{array}\right] \\
& C=\left[\begin{array}{cccccc}
c_{1,1}-\rho_{0} \cos \varphi \exp \left(\alpha_{0} V_{2} \cos \varphi\right) & c_{2,1}+\rho_{0} \sin \varphi \exp \left(\alpha_{0} V_{2} \cos \varphi\right) & 0 & -1 & 0 & 0 \\
c_{1,2}+\rho_{0} \exp \left(\alpha_{0} V_{2} \sin \varphi\right) & c_{2,2}+\rho_{0} \operatorname{ctg} \varphi \exp \left(\alpha_{0} V_{2} \sin \varphi\right) & 0 & 0 & -1 & 0 \\
-k_{T} \rho_{0}\left\{\cos \varphi \exp \left(\alpha_{0} V_{2} \cos \varphi\right)+\right. & k_{T} \rho_{0}\left\{\sin \varphi \exp \left(\alpha_{0} V_{2} \cos \varphi\right)+\right. & & & & \\
\left.+\exp \left(\alpha_{0} V_{2} \sin \varphi\right)\right\} & \left.+\operatorname{ctg} \varphi \exp \left(\alpha_{0} V_{2} \sin \varphi\right)\right\} & c_{3,3} & 0 & 0 & -1 \\
-\alpha \chi_{1} & \beta \chi_{1} & 0 & 1 & 0 & 0 \\
-\alpha \chi_{2} & \beta \chi_{2} & 0 & 0 & 1 & 0 \\
-\alpha \chi_{3} & \beta \chi_{3} & 0 & 0 & 0 & 1
\end{array}\right] ; \\
& \alpha=\rho\left[\left[1+\mu \exp \left(-\zeta V_{3}\right)\right]\left[t_{P}^{(0)} \sin \varphi-S_{P}^{(0)} \cos \varphi-\left(X_{1}^{*} \cos \varphi+X_{2}^{*} \sin \varphi\right) \sin \varphi\right] ;\right. \\
& \beta=\rho\left[\left[1+\mu \exp \left(-\zeta V_{3}\right)\right]\left[t_{P}^{(0)} \cos \varphi+S_{P}^{(0)} \sin \varphi-\left(X_{l}^{*} \sin \varphi+X_{2}^{*} \cos \varphi\right) \cos \varphi\right] ;\right. \\
& \varsigma=\rho \mu \zeta \exp \left(-\zeta V_{3}\right) S_{P}^{(0)}\left[t_{P}^{(0)}-\left(X_{1}^{*} \cos \varphi+X_{2}^{*} \sin \varphi\right) \sin \varphi\right] ; t_{P, \Sigma}=t_{P}^{(0)}-\left(X_{1}^{*} \sin \varphi+X_{2}^{*} \cos \varphi\right) \text {. }
\end{aligned}
$$


For studying of the agreement of the controlling trajectories with dynamic properties of the cutting system the many numeric experiments are done and it's the main result is represented below.

\section{Results}

The analyze (7) is showed that the stability depends on $t_{P}^{(0)}, S_{P}^{(0)}$ and speeds $V_{2}, V_{3}$ which are determined by trajectories of the space $\mathfrak{R}_{Z}^{(3)}$. Consequently, it is necessary to choose the trajectories are meeting the requirement to stability from the $Z$ trajectories are meeting the setting requirements to accuracy of the detail. Besides that, the variation of the technological regimes leads to change of some system parameters. Here the first of all it is necessary to specify to decrease of the time constant $T_{i}, i=1,2,3$ with increase of the cutting speed. Here as a rule there is a limited range of the speed in which the system is stability. The cutting depth $t_{P}^{(0)}$ and the shaving pressure on the front tool surface $\rho$ principal influence on the stability. Their increase always promotes to loss of the stability. It should be noted that all these parameters depend on the trajectories of the machine executive elements which actually play role of the controlling parameters in the dynamic cutting system.

Besides we have parameters that don't depend on the trajectories of the space $\mathfrak{R}_{Z}^{(3)}$. It is the first of all corners $\varphi, \alpha$ and $\gamma$ (fig. 1). They can be varied on the stage of the preparation of the technological process. The influence of the corner $\varphi \leq \pi / 2$ is considered in beginning. With his decrease the length of the contact of the tool cutting edge increase and consequently $\partial F_{i} / \partial x_{i}$. Therefore, the tendency to loss of the stability is increase. If the corner $\varphi$ match with orientation of the rigidity ellipsoid of the tool subsystem in plane $Y_{1}, Y_{2}$ then the results of modeling are showed that the increase of the stability margin are observed because of the scalarization of the elastic properties of the tool subsystem. Therefore, there is the optimum value of corner $\varphi$. The change of the tool geometry (the first of all the corner $\gamma$ ) influences on the corner's coefficients $\chi_{i}, i=1,2,3$. As a rule with increase this corner the projections of the force decrease in directional of the release from cutting zone. Therefore, the stability margin increase. The increase of the corner $\gamma$ causes the decrease of the shaving pressure on the tool surface, leads to increase stability margin. However here it is necessary to compromise between the stability margin and decrease of durability of the cutting edge of the tool. At last the back corner $\alpha>0$ with small variations in the equilibrium vicinity practically does not impact on the stability. However in case when the cutting system has the additional perturbations for example the spindle beats group then the additional effect of the self-excitation of the cutting system is observed at small value of this corner.

\section{Conclusion}

It is necessary to provide the agreement of the CNC program and the trajectories of the movement of the executive elements of the machine during controlling of the cutting process on the machine but and these trajectories agree with dynamic properties of the cutting system. The provided data show that one of conditions of agreement is the choice of 
the geometry parameters of the tool in which the controlling trajectories are asymptotically stability.

\section{References}

1. G. Haken, Secrets of the nature. Synergetic: the doctrine of interaction (Institute of the computer researches, Moscow-Izhevsk, 2003)

2. G. Haken, Synergetic (Mir, Moscow, 1980)

3. I. Prigogine, From being to becoming (Science, Moscow, 1985)

4. I. Prigogine, I. Stengers, Order out of chaos: Man's new dialogue with the nature (Progress, Moscow, 1986)

5. A.A. Kolesnikov, Synergetics method of control of complex system: theory of the system analisys (DomKniga, Moscow, 2006)

6. A.A. Kolesnikov, Synergetics and control theory problems (Fizmatlit, Moscow, 2004)

7. V.L. Zakovorotny, M.B. Flek, Dynamics of Cutting Process: Synergetic Approach, (Terra, Rostov-on-Don, 2006)

8. V.L. Zakovorotny, A.D. Luk'yanov, D.-A. Nguen, D.T. Fam, Synergetic System Synthesis of Controlled Dynamics of Metal Cutting Machines Takinginto Account the Evolution of Relations (Donsk. Gos. Tekh. Univ., Rostov-on-Don, 2008)

9. V.L. Sosonkiy, G.M. Martinov, The latest tendencies in the field of architectural concepts of the CNC systems. Automation in the industry, 4, 3-9 (2005)

10. G.M. Martinov, N.V. Kozak, R.A. Nezhmetdinov, A.S. Grigoriev, A.I. Obukhov, L.I. Martinova, Autom. Remote Control, 78 (3), 525 (2017)

11. V.L. Zakovorotny, V.P. Lapshin, I.A. Turkin, Scientific-educational and applied journal. University News. North-Caucasian region. Technical Sciences Series, 3 (178), 33 (2014)

12. V.L. Zakovorotny, E.Y. Panov, P.N. Potapenko, Vestnik of DSTU, 1(2) (2001)

13. V.L. Zakovorotny, T. Sankar, E.V. Borodachev, STIN, 22, 12 (1994)

14. V.L. Zakovorotny, A.D. Lukyanov, Int. J. of Mech. Eng. and Auto, 1(5), 271 (2014)

15. V.L. Zakovorotny, M.F. Flek, Pham Dinh Tung, Vestnik of DSTU, 11, 1785 (2011)

16. V.L. Zakovorotny, D.T. Pham, S.T. Nguen, M.N. Rigkin, Vestnik DSTU, 11, 137 (2011)

17. V.L. Zakovorotny, D.T. Fam, S.T. Nguen, M.N. Rigkin, Vestnik DSTU, 11, 301 (2011)

18. V.L. Zakovorotny, V.E. Gvindjiliya, News of h. educ. Inst. App. nonlinear dyn., 25, 40 (2017)

19. L.S. Pontrygin, Izbrannyye nauchnyye trudy, 2, 95-154 (Science, Moscow, 1998)

20. A. N. Tikhonov, Mathematical collection, 31, 231 (1951)

21. A.N. Lypunov, A.M. Lypunov, The general task of the stability of the movement, (Gostechizdat, Moscow, 1950) 